(C) Revista de Matemática: Teoría y Aplicaciones 2022 29(1) : 19-37

CIMPA - UCR ISSN: 1409-2433 (PRINT), 2215-3373 (ONLINE)

DOI: https://doi.org/10.15517/rmta.v29i1.41404

\title{
OPERADS LIBRES SOBRE MÓDULOS DIFERENCIALES GRADUADOS
}

\section{FREE OPERADS IN DIFFERENTIAL GRADED MODULES}

\author{
JESÚS SÁNCHEZ-GUEVARA*
}

Received: 7/Aug/2020; Revised: 4/Oct/2021;

Accepted: 17/Nov/2021

\begin{abstract}
Revista de Matemática: Teoría y Aplicaciones is licensed under a Creative Commons Attribution-NonCommercial-ShareAlike 4.0 International License.

http://creativecommons.org/licenses/by-nc-sa/4.0/
\end{abstract}

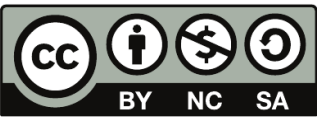

*Universidad de Costa Rica, Centro de Investigación en Matemática Pura y Aplicada (CIMPA) y Escuela de Matemáticas, San José, Costa Rica. E-Mail: jesus.sanchez_g@ucr.ac.cr 


\title{
Resumen
}

Los operads son estructuras algebraicas que han manifestado su importancia en el estudio y clasificación de las propiedades homotópicas de espacios de lazos. Este artículo aborda el estudio de la noción de operad libre, tanto para el caso simétrico, como para el caso no simétrico, pues este tipo de construcción representa un método valioso en el diseño de operads en diferentes situaciones. Para hacer esto, los operads simétricos son interpretados como monoides sobre la categoría de $\mathbb{S}$-módulos. Este trabajo tiene como objetivo mostrar algunas de las propiedades principales entre los funtores asociados a las construcciones de operads simétricos libres para la comprensión de los mecanismos de este tipo de estructuras. Lo cual lleva al resultado principal de este artículo, donde se expresa al funtor de operads libres simétricos en términos del funtor de operads libres no simétricos cuando las acciones de los grupos simétricos son libres.

Palabras clave: operads; módulos diferenciales graduados; funtor de operads libres.

\begin{abstract}
Operads are algebraic structures who have manifested their importance in the study and classification of the homotopic properties of loop spaces. This paper makes a survey of the notion of free operad, both for the symmetric case and for the non-symmetric case, since this type of construction represents a valuable method in the design of operads in different situations. In order to do this, the symmetric operads are interpreted as monoids on the category of $\mathbb{S}$-modules. This work has as objective to show some of the main properties between the functors associated with the constructions of free symmetric operads for understanding the mechanisms of this type of structure. Which leads to the main result of this paper, where the symmetric free operad functor is expressed in terms of the non-symmetric free operad functor, when the actions by the symmetric groups are free.
\end{abstract}

Keywords: operads; differential graded modules; free operads functor.

Mathematics Subject Classification: 18N70; 55U15

\section{Introducción}

La noción de operad aparece en los años sesenta cuando James Dillon Stasheff usa $A_{\infty}$-álgebras para estudiar estructuras algebraicas que tienen propiedades asociativas no estrictas ([11]), es decir, que se cumplen bajo equivalencias de homotopía. Luego, con el fin de describir la estructura homotópica subyacente a los espacios de lazos, Peter May introduce en los inicios de los años setenta 
el concepto de operad ([5]), el cual, entre otras cosas, engloba las álgebras estudiadas por Stasheff. En su trabajo, May demuestra que, si un espacio tiene una estructura de álgebra sobre ciertos tipos de operads, entonces este espacio es homotópicamente equivalente a un espacio de lazos, iterado finita o infinitamente.

Intuitivamente, el uso de un operad puede verse como una generalización de la relación entre un grupo y sus representaciones, pero con la diferencia de que la estructura algebraica a describir puede que tenga muchas operaciones de diferentes aridades y que estas operaciones no necesariamente cumplan con condiciones de conmutatividad o asociatividad. Así, un operad $\mathcal{P}$ está conformado por diferentes tipos de objetos, cuya naturaleza depende de la categoría en la que se esté trabajando. Estos objetos son los que organizan las operaciones abstractas que luego serán realizadas como las operaciones concretas a describir. Por ejemplo, $P(m)$ representaría las operaciones de nuestra entidad que tienen aridad $m$ o $m$ entradas, y una salida. Las condiciones que se le piden a estas familias de operaciones son, que cuenten con una composición asociativa y con un elemento neutro con respecto a la composición. En el caso de los operads simétricos, también se necesitan condiciones de equivarianza con respecto a las acciones de los grupos simétricos.

Para efectos de este artículo, la teoría de operads será estudiada en el marco de las categorías de módulos diferenciales graduados y se utilizará como punto de partida la definición clásica de operads simétricos formulada por May. Luego, se abordará de forma detallada el estudio de una construcción de operads libres para el caso simétrico, la cual se puede interpretar como una generalización de las estructuras libres usuales asociadas a grupos, anillos, espacios vectoriales, etc. Además, luego de unas pocas adaptaciones, se verá como dicha construcción también funciona para el caso de operads no simétricos.

Este análisis minucioso describe el punto de vista categórico de la noción de operads, donde los operads son descritos como cierto tipo de monoides. Lo cual se puede considerar como una construcción de operads simétricos libres más cercana a la intuición, pues con los productos tensoriales asociados, las interpretaciones de los objetos, como operaciones de múltiples entradas, son más claras.

El interés en abordar este tipo de enfoque radica en la descripción de las relaciones entre los diferentes funtores asociados a las construcciones de operads libres para los casos simétricos y no simétricos, recopiladas en el teorema 5.9. Lo cual nos lleva al resultado principal de este artículo, concretizado como el corolario 5.12, donde se ve la relación entre las construcciones de operadas libres simétricos y no simétricos, al expresar el funtor de operads libres del caso simétrico en términos de su homólogo del caso no simétrico, cuando las acciones 
simétricas sobre los objetos son libres. Este artículo presenta lo descrito anteriormente de la siguiente manera. En la sección 2 se recuerdan las generalidades de la teoría de la categoría de módulos diferenciales graduados, los cuales determinan el marco sobre el cual los operads de este artículo son construidos. En la sección 3 se introducen la categoría de operads simétricos y se ven algunos ejemplos clásicos. En la sección 4 se hace un estudio detallado de la construcción de operads simétricos libres, la cual es posible al redefinir los operads simétricos como monoides en la categoría de $\mathbb{S}$-módulos. Finalmente, en la sección 5 se realiza la construcción de operads libres para el caso no simétrico junto con el planteamiento de las relaciones entre los funtores asociados de ambas construcciones, y se expone el resultado principal de este artículo, el cual describe el funtor de operads libres simétricos a través del caso no simétrico.

El presente trabajo está basado en el segundo capítulo de la tesis doctoral del autor ([10]), donde esta manera de abordar la construcción de operads libres lleva al diseño de operads del tipo $E_{\infty}$, los cuales son usados para estudiar las propiedades homotópicas de estructuras asociadas a complejos de cadenas, como las descritas por Alain Prouté en [6] y [7].

\section{Preliminares}

Se utilizarán libremente el lenguaje y las propiedades de los módulos diferenciales graduados que aparecen en [2] y [10]. Con respecto a la teoría de categorías, las terminologías y propiedades son tomadas de [3] y [8].

Usaremos $F$ para denotar un cuerpo, el cual puede ser $\mathbb{Z} / p \mathbb{Z}$, donde $p$ es un número primo, o incluso $\mathbb{Q}$. Los módulos sobre $F$ serán llamados simplemente módulos. Se denota $[n]$ al conjunto $\{1, \ldots, n\}$, donde $n$ es un entero positivo. El grupo simétrico formado por las permutaciones de $[n]$ se escribe $\Sigma_{n}$.

Un módulo $M$ se dirá módulo graduado si existe una familia $\left\{M_{i}\right\}_{i \in \mathbb{Z}}$ de submódulos de $M$, tales que $M=\bigoplus_{i \in \mathbb{Z}} M_{i}$. Un módulo diferencial graduado o DG-módulo es un módulo graduado $M$ junto con un morfismo $\partial: M \rightarrow M$ de grado -1 , tal que $\partial \circ \partial=0$. Una aumentación de un DG-módulo es un morfismo de DG-módulos $\epsilon: M \rightarrow F$ de grado 0. Similarmente, una coaumentación de $M$ es un morfismo de DG-módulos $\eta: F \rightarrow M$ de grado 0. Si un DG-módulo $M$ tiene una aumentación $\epsilon$ y una coaumentación $\eta$, tal que $\epsilon \circ \eta=1_{F}$, entonces $M$ se dice módulo diferencial graduado con aumentación o DGA-módulo. Se denota la categoría de DGA-módulos como DGA-Mod.

Debido a que se trabajará en un contexto graduado, los signos de los diagramas incluidos están determinados por la convención de Koszul y son omitidos para facilitar la lectura. Recuerde que la convención de Koszul dice que, si la 
posición de dos símbolos en una expresión, de grados $p$ y $q$, es permutada, la expresión resultante será multiplicada por $(-1)^{p q}$.

\section{Operads simétricos}

Los operads simétricos son colecciones de objetos que tienen asociados a sus componentes acciones de los grupos simétricos $\Sigma_{n}$.

Definición 3.1 Un operad simétrico $\mathcal{P}$ es una colección de DGA-módulos $\{\mathcal{P}(n)\}_{n \geqslant 0}$ junto con:

1. Un morfismo $\eta: F \rightarrow \mathcal{P}(1)$, llamado la unidad de $\mathcal{P}$.

2. Para cada $n$, una acción a la derecha por el grupo simétrico $\Sigma_{n}$ sobre $\mathcal{P}(n)$, es decir, un morfismo de DGA-módulos que hace de $P(n)$ un DGA$F\left[\Sigma_{n}\right]$-módulo derecho.

$$
P(n) \otimes F\left[\Sigma_{n}\right] \longrightarrow P(n)
$$

3. Por cada tupla $\left(h, i_{1}, \ldots, i_{h}\right)$, un morfismo de DGA-módulos,

$$
\gamma_{\left(h, i_{1}, \ldots, i_{h}\right)}: \mathcal{P}(h) \otimes \mathcal{P}\left(i_{1}\right) \otimes \cdots \otimes \mathcal{P}\left(i_{h}\right) \rightarrow \mathcal{P}(n)
$$

donde $n=i_{1}+\cdots+i_{h}$ y $n, h, i_{j} \geqslant 0$. Todo morfismo de este tipo se escribirá $\gamma$.

Estos morfismos deben de cumplir las siguientes condiciones.

1. Los morfismos $\gamma$ son asociativos, en el sentido del siguiente diagrama conmutativo:

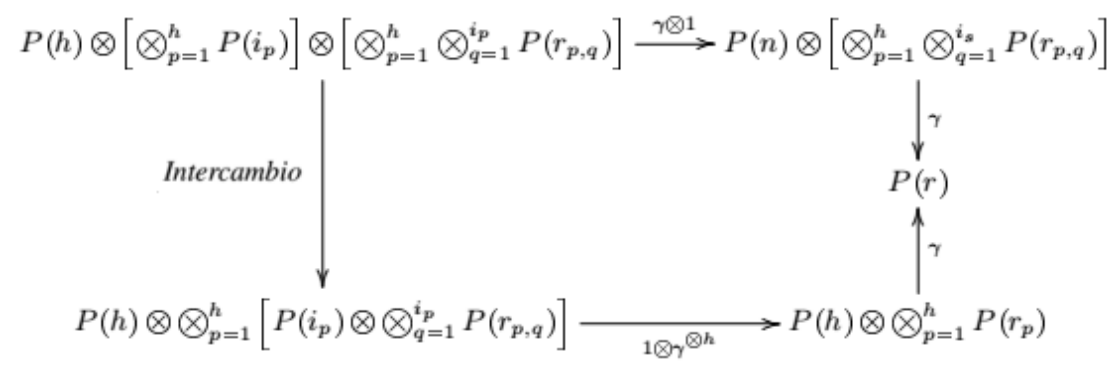

donde $n=\sum_{p=1}^{h} i_{p}, r=\sum_{p=1}^{h} \sum_{q=1}^{i_{p}} r_{p, q}=\sum_{p=1}^{h} r_{p}$ y la flecha vertical izquierda es solamente un intercambio de factores.

Rev.Mate.Teor.Aplic. (ISSN print: 1409-2433; online: 2215-3373) Vol. 29(1): 19-37, Jan-Jun 2022 
2. La unidad $\eta: F \rightarrow P(1)$ hace conmutativos los siguientes diagramas.

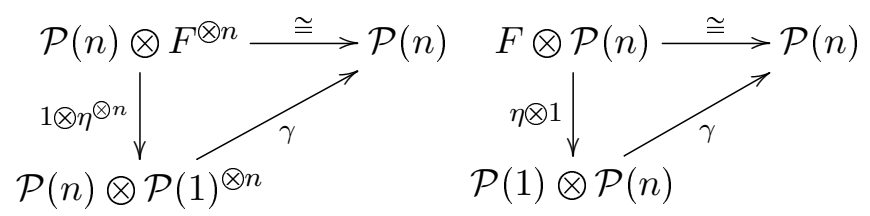

3. Las acciones de los grupos simétricos deben satisfacer condiciones de equivarianza, expresadas por los siguientes diagramas conmutativos.

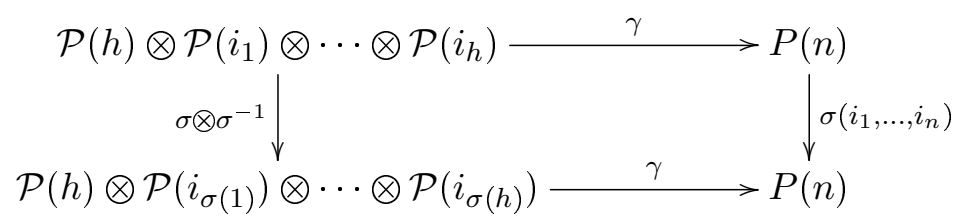

donde $n=i_{1}+\cdots+i_{h}$ y la flecha $\sigma \otimes \sigma^{-1}$ consiste en la acción derecha de $\sigma$ sobre $P(h)$ y la acción izquierda de $\sigma^{-1}$ sobre el producto tensorial $P\left(i_{1}\right) \otimes \cdots \otimes P\left(i_{h}\right)$.

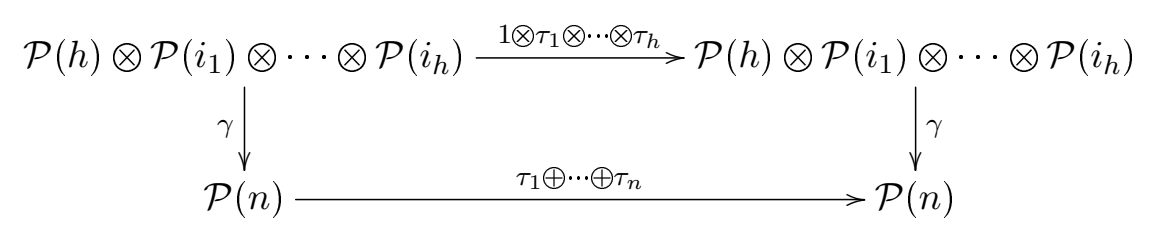

donde $n=i_{1}+\cdots+i_{h}$ y la acción $1 \otimes \tau_{1} \otimes \cdots \otimes \tau_{h}$ es la identidad de $P(h)$ sobre el primer factor y la acción derecha de $\tau_{j}$ en el factor $P\left(i_{j}\right)$.

El operad de endomorfismos ser puede ver como el paradigma para diseñar el concepto de operad simétrico.

Definición 3.2 Para cada $M \in D G A-M o d$, el operad simétrico End(M), llamado operad de endomorfismos de $M$, se define como:

1. Para todo $n \geqslant 0, \operatorname{End}(M)(n)=H o m\left(M^{\otimes n}, M\right)$, es decir el DGAmódulo de aplicaciones homogéneas de $M^{\otimes n}$ a $M$.

2. La unidad $\eta: F \rightarrow \operatorname{End}(M)(1)$ es la identidad de $M$.

3. La acción derecha de $\Sigma_{n}$ sobre End( $\left.M\right)$ es inducida por la acción izquierda de $\Sigma_{n}$ sobre $M^{\otimes n}$, es decir, $f \sigma\left(x_{1} \otimes \cdots \otimes x_{n}\right)=\|\sigma\| f\left(x_{\sigma^{-1}(1)} \otimes \cdots \otimes\right.$ $\left.x_{\sigma^{-1}(n)}\right)$. Donde $\|\sigma\|$ es el signo de la permutación $\sigma$. 
4. La composición de aplicaciones,

$\gamma: \operatorname{End}(M)(h) \otimes \operatorname{End}(M)\left(i_{1}\right) \otimes \cdots \otimes \operatorname{End}(M)\left(i_{h}\right) \rightarrow \operatorname{End}(M)(n)$

donde $n=i_{1}+\cdots+i_{h}$, está dada por,

$$
\gamma\left(f_{h} \otimes f_{i_{1}} \otimes \cdots \otimes f_{i_{h}}\right)=f_{h} \circ\left(f_{i_{1}} \otimes \cdots \otimes f_{i_{h}}\right)
$$

La verificación que $\operatorname{End}(M)$ satisface las condiciones de la definición 3.1 es inmediata.

Definición 3.3 Sean $P$ y $Q$ dos operads simétricos. Un morfismo $f$ de $P$ a $Q$, es una colección de morfismos de DGA-módulos,

$$
f_{n}: P(n) \rightarrow Q(n)
$$

que satisfacen las siguientes condiciones.

1. El morfismo $f_{1}: P(1) \rightarrow Q(1)$ preserva la unidad de los operads simétricos, es decir, $f_{1} \eta=\eta$.

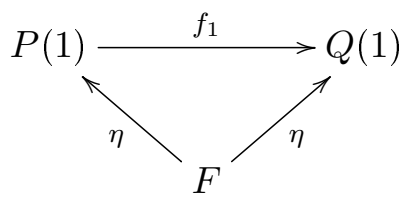

2. Los morfismos $f_{n}: P(n) \rightarrow Q(n)$ son $\Sigma_{n}$-equivariantes, es decir, el siguiente diagrama es conmutativo para cada $\sigma \in \Sigma_{n}$.

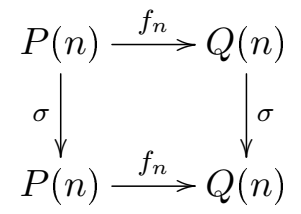

3. f preserva las operaciones de composición de los operads simétricos, esto es, que el siguiente diagrama es conmutativo.

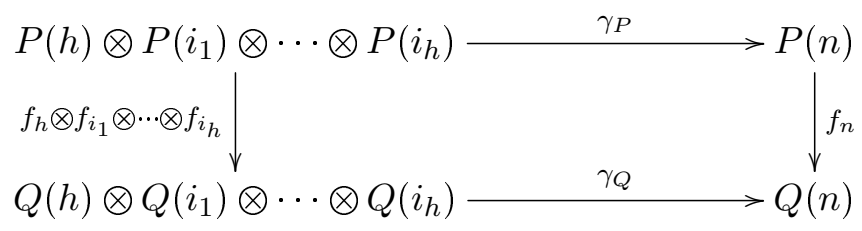


La categoría de operads simétricos sobre DGA-Mod se denota $\mathcal{O P}$.

Ejemplo 3.4 El operad simétrico $\mathcal{N}$ está dado por $\mathcal{N}(n)=F$ para cada $n$ no negativo, donde $F$ es visto como un DGA-módulo concentrado en grado cero. La unidad $\eta$ es la identidad de $F, \Sigma_{n}$ actúa de manera trivial en cada componente y las composiciones $\gamma$, si denotamos $a_{i}$ al generador de grado cero de $N(i)$, están dadas por la regla,

$$
\gamma: a_{h} \otimes a_{i_{1}} \otimes \cdots \otimes a_{i_{h}} \rightarrow a_{n}
$$

donde $n=i_{1}+\cdots+i_{h}$.

Ejemplo 3.5 Al hacer libre la acción de los grupos simétricos en el ejemplo anterior, se genera un operad simétrico que denotamos $\mathcal{M}$. Las componentes de $\mathcal{M}$ son los módulos concentrados en grado cero $M(n)=F\left[\Sigma_{n}\right]$, para cada $n$ no negativo. Las composiciones $\gamma$ son determinadas, al igual que antes, por los generadores de grado cero, respetando las acciones de los grupos simétricos:

$$
\gamma\left(a_{h} \otimes a_{i_{1}} \sigma_{i_{1}} \otimes \cdots \otimes a_{i_{h}} \sigma_{i_{1}}\right)=a_{n}\left(\sigma_{i_{1}} \oplus \cdots \oplus \sigma_{i_{h}}\right)
$$

$y$

$$
\gamma\left(a_{h} \sigma \otimes a_{i_{\sigma^{-1}(1)}} \otimes \cdots \otimes a_{i_{\sigma^{-1}(h)}}\right)=a_{n} \sigma\left(i_{1}, \ldots, i_{h}\right)
$$

donde $n=i_{1}+\cdots+i_{h}$.

\section{Operads simétricos libres}

Al dejar de lado la composición en un operad simétrico $\mathcal{P}$, lo que resta es una colección $\{P(n)\}_{n \geqslant 0}$ de DGA-módulos con acciones a la derecha por los grupos simétricos respectivos. Las colecciones de este tipo las llamaremos $\mathbb{S}$-módulos. En esta sección veremos una manera de generar a partir de un $\mathbb{S}$-módulo, una estructura de operad simétrico que lo contenga. Los operads simétricos obtenidos de esta forma son llamados operads simétricos libres y van a satisfacer la siguiente propiedad universal: cada morfismo de $\mathbb{S}$-módulos, entre la colección de partida a cualquier otra colección con una estructura de operad simétrico, puede ser extendido de manera única a un morfismo de operads simétricos desde el operad simétrico libre.

La construcción de operads simétricos libres es descrita en las principales referencias del tema, por ejemplo: [2], [4], [1] y [9]. Para ello, los operads simétricos son definidos como monads sobre la categoría de endofuntores de la categoría de DGA-módulos y donde los $\mathbb{S}$-módulos son identificados con funtores de Schur (ver [2]). 
Sin embargo, es posible describir esta construcción con técnicas similares, pero evitando el uso de de los funtores de Schur, lo cual es el principal objetivo de esta sección. Así, para obtener un operad simétrico libre a partir de un S-módulo, los operads simétricos se puede interpretar como monoides sobre la categoría de $\mathbb{S}$-módulos, donde el producto monoidal se busca que codifique la composición generalizada de operaciones abstractas. De esta forma, los $\mathbb{S}$-módulos se mantienen en su estado natural y la composición de funtores de Schur aparecerá como una operación especial de $\mathbb{S}$-módulos.

Aunque hay muchas formas de definir operads simétricos libres, con esta forma de presentar su construcción, los operads simétricos se pueden mantener más cerca del punto de vista clásico de la definición 3.1. Este enfoque se puede usar, entre otras cosas, para facilitar el diseño de algunos tipos de $E_{\infty}$-coalgebras, las cuales son coalgebras sobre un operad simétrico del tipo $E_{\infty}$ (ver [10]).

Definición 4.1 Sea $\mathbb{S}$ el grupoide donde los objetos son los conjuntos ordenados $[n]=\{1, \ldots, n\}$, donde $n$ es un entero positivo y $[0]=\varnothing$. Los morfismos de $\mathbb{S}$ están dados por $\mathbb{S}(n, m)=\varnothing$, si $n \neq m, y \mathbb{S}(n, n)=\Sigma_{n}$, el $n$ grupo simétrico.

Definición 4.2 Un $\mathbb{S}$-módulo $M$ es un funtor contravariante de la categoría $\mathbb{S}$ a la categoría DGA-Mod. Los morfismos $\mathbb{S}(n, n)$ son interpretados como acciones a la derecha de $\Sigma_{n}$ sobre $M(n)$. La categoría de $\mathbb{S}$-módulos y transformaciones naturales se denota $\mathbb{S}-M o d$.

Observe que la categoría $\mathbb{S}-$ Mod tiene todos los colímites y límites debido a que es una categoría de diagramas sobre DGA-Mod.

Definición 4.3 Se denota U el funtor de olvido de la categoría de operads simétricos a la categoría $\mathbb{S}-$ Mod.

Antes de la construcción del funtor que define los operads simétricos libres sobre $\mathbb{S}-M o d$, veamos en detalle como luce un operad simétrico libre. Sea $M$ un S-módulo, si para cada entero $n \geqslant 1$, su componente $M(n)$ se piensa como constituido por aplicaciones de $n$ entradas y una salida, entonces el operad simétrico libre $F(M)$ asociado a $M$ se puede entender como todas las posibles aplicaciones que se obtienen al componer formalmente los elementos de $M$. Es claro que, como $\mathbb{S}$-módulo, $F(M)$ deberá contener a $M$ como un $\mathbb{S}$-submódulo.

Así, la componente $F(M)(n)$ contendrá a $M(n)$ y además, deberá contener a todos los posibles productos tensoriales del tipo $M(h) \otimes M\left(i_{1}\right) \otimes \cdots \otimes M\left(i_{h}\right)$, donde $i_{1}+\cdots+i_{h}=n$, ya que ellos representan las composiciones formales que dan como resultado operaciones de aridad $n$. 
Para satisfacer el axioma de equivarianza (5) necesitamos, para cada $\sigma \in \Sigma_{h}$, la relación,

$$
M(h) \sigma \otimes M\left(i_{1}\right) \otimes \cdots \otimes M\left(i_{h}\right)=M(h) \otimes M\left(i_{\sigma(1)}\right) \otimes \cdots \otimes M\left(i_{\sigma(h)}\right)
$$

la cual se obtiene tomando el producto tensorial sobre $F\left[\Sigma_{h}\right]$,

$$
M(h) \otimes_{\Sigma_{h}} M\left(i_{1}\right) \otimes \cdots \otimes M\left(i_{h}\right) .
$$

Ahora, considere el segundo axioma de equivarianza (6). En la parte derecha de $(17), M\left(i_{1}\right) \otimes \cdots \otimes M\left(i_{h}\right)$, podríamos tener acciones sobre cada factor por elementos del respectivo grupo simétrico,

$$
M\left(i_{1}\right) \tau_{1} \otimes \cdots \otimes M\left(i_{h}\right) \tau_{h}, \text { donde } \tau_{j} \in \Sigma_{i_{j}}
$$

La acción simultánea de las permutaciones $\tau_{j}$ puede verse como una acción de la permutación de $\Sigma_{n}$ dada por $\tau_{1} \oplus \cdots \oplus \tau_{k}$, actuando a la derecha de $M\left(i_{1}\right) \otimes$ $\cdots \otimes M\left(i_{h}\right)$. Las permutaciones de este tipo forman el subgrupo $\Sigma_{i_{1}} \times \cdots \times \Sigma_{i_{h}}$ de $\Sigma_{n}$. Entonces ponemos escribir,

$$
M\left(i_{1}\right) \tau_{1} \otimes \cdots \otimes M\left(i_{h}\right) \tau_{h}=\left(M\left(i_{1}\right) \otimes \cdots \otimes M\left(i_{h}\right)\right)\left(\tau_{1} \oplus \cdots \oplus \tau_{h}\right)
$$

El proceso de colocar a la derecha las permutaciones $\tau_{j}$ es entonces expresado por el producto tensorial,

$$
\left(M\left(i_{1}\right) \otimes \cdots \otimes M\left(i_{h}\right)\right) \otimes \Sigma_{i_{1} \times \cdots \times \Sigma_{i_{h}}} F\left[\Sigma_{n}\right]
$$

En esta expresión se coloca $F\left[\Sigma_{n}\right]$ en lugar de $F\left[\Sigma_{i_{1}} \times \cdots \times \Sigma_{i_{h}}\right]$, para así considerar todas las otras permutaciones de $\Sigma_{n}$ que actúan sobre $i_{1}+\cdots+i_{h}$ entradas pero no pueden ser expresadas por una suma del tipo $\tau_{1} \oplus \cdots \oplus \tau_{h}$. Además, esto se simplifica escribiendo,

$$
\left(M\left(i_{1}\right) \otimes \cdots \otimes M\left(i_{h}\right)\right) \otimes F\left[\Sigma_{n} /\left(\Sigma_{i_{1}} \times \cdots \times \Sigma_{i_{h}}\right)\right]
$$

El cociente $\Sigma_{n} /\left(\Sigma_{i_{1}} \times \cdots \times \Sigma_{i_{h}}\right)$ es el grupo de los $\left(i_{1}, \ldots, i_{h}\right)$ intercambios de $\Sigma_{n}$, el cual se denota $S h\left(i_{1}, \ldots, i_{h}\right)$. Recuerde que un $\left(i_{1}, \ldots, i_{h}\right)$ intercambio, donde $i_{1}+\cdots+i_{h}=n$, es un elemento de $\Sigma_{n}$ que envía $(1, \ldots, n)$ a $\left(\mu_{1}^{1}, \ldots, \mu_{i_{1}}^{1}, \ldots, \mu_{1}^{h}, \ldots, \mu_{i_{h}}^{h}\right)$ tal que $\mu_{1}^{j}<\ldots<\mu_{i_{j}}^{j}$ para todo $1 \leqslant j \leqslant h$. Así, (21) se escribe,

$$
M\left(i_{1}\right) \otimes \cdots \otimes M\left(i_{h}\right) \otimes F\left[S h\left(i_{1}, \ldots, i_{h}\right)\right]
$$

El cual junto con la parte $M(h)$ nos da la siguiente expresión.

$$
M(h) \otimes \Sigma_{h} M\left(i_{1}\right) \otimes \cdots \otimes M\left(i_{h}\right) \otimes F\left[S h\left(i_{1}, \ldots, i_{h}\right)\right]
$$


Nuestro operad simétrico libre necesitará esta construcción para cualquier $n$ y todas las posibles sumas $i_{1} \cdots+i_{h}=n$, es decir, necesitamos considerar la suma directa,

$$
\bigoplus_{n \geqslant 0} \bigoplus_{h \geqslant 0} M(h) \otimes \Sigma_{h}\left(\underset{i_{1}+\cdots+i_{h}=n}{\bigoplus_{1}} M\left(i_{1}\right) \otimes \cdots \otimes M\left(i_{h}\right) \otimes F\left[S h\left(i_{1}, \ldots, i_{h}\right)\right]\right)
$$

Esta expresión representa la primera etapa de todas las posibles composiciones formales entre los elementos de $M$ cuando son interpretados como aplicaciones. En la siguiente etapa de composiciones se deben de considerar cuando cada $M\left(i_{j}\right)$ en 24 viene de otra composición arbitraria y así sucesivamente, una cantidad finita de pasos. Para poder manejar todas los posibles niveles de composiciones necesitamos introducir algunas operaciones sobre los $\mathbb{S}$-módulos.

Definición 4.4 Sean $M$ y $N \mathbb{S}$-módulos. Se define el producto tensorial de $M y$ $N$ como el $\mathbb{S}$-módulo $M \otimes N$ dado por la fórmula,

$$
(M \otimes N)(n)=\bigoplus_{i+j=n} M(i) \otimes M(j) \otimes F[S h(i, j)]
$$

Proposición 4.5 El producto tensorial de $\mathbb{S}$-módulos es asociativo y para cada $\mathbb{S}$-módulo $M$ satisface $M=M \otimes F=F \otimes M$, donde $F$ se ve como un $\mathbb{S}$-módulo concentrado en aridad 0.

Demostración. Sean $M, N$ y $P$, S-módulos.

$$
\begin{aligned}
& ((M \otimes N) \otimes P)(n)=\bigoplus_{i+j=n}(M \otimes N)(i) \otimes P(j) \otimes F[S h(i, j)] \\
& \quad=\bigoplus_{i+j=n} \bigoplus_{r+s=i} M(r) \otimes N(s) \otimes F[S h(r, s)] \otimes P(j) \otimes F[S h(i, j)] \\
& =\bigoplus_{i+j=n} \bigoplus_{r+s=i}\left(M(r) \otimes N(s) \otimes \Sigma_{r} \times \Sigma_{s} F\left[\Sigma_{i}\right]\right) \otimes \Sigma_{i} \times \Sigma_{j} P(j) \otimes F\left[\Sigma_{n}\right] \\
& =\bigoplus_{r+s+j=n} M(r) \otimes N(s) \otimes P(j) \otimes \Sigma_{r} \times \Sigma_{s} \times \Sigma_{j} F\left[\Sigma_{n}\right] \\
& =\bigoplus_{r+i=n s+j=i} \bigoplus_{s} M(r) \otimes\left(N(s) \otimes P(j) \otimes_{\Sigma_{s} \times \Sigma_{j}} F\left[\Sigma_{i}\right]\right) \otimes \Sigma_{r} \times \Sigma_{i} F\left[\Sigma_{n}\right] \\
& =(M \otimes(N \otimes P))(n)
\end{aligned}
$$

El resto de la prueba es directa.

Obervación 4.6 Note que en la fórmula 24) se tiene,

$$
\bigoplus_{i_{1}+\cdots+i_{h}=n} M\left(i_{1}\right) \otimes \cdots \otimes M\left(i_{h}\right) \otimes F\left[S h\left(i_{1}, \ldots, i_{h}\right)\right]=M^{\otimes h}
$$


donde $M^{\otimes h}$ es $h$ veces el producto tensorial de $\mathbb{S}$-módulos.

Definición 4.7 Sean $M$ y $N$ S-módulos. Se define la composición de $M$ con $N$ como el $\mathbb{S}-m o ́ d u l o$,

$$
M \circ N=\bigoplus_{h \geqslant 0} M(h) \otimes_{\Sigma_{h}} N^{\otimes h}
$$

Obervación 4.8 La fórmula (24) puede ser escrita,

$$
\begin{aligned}
& \bigoplus_{h \geqslant 0} M(h) \otimes \Sigma_{h}\left(\bigoplus_{n \geqslant 0} \bigoplus_{i_{1}+\cdots+i_{h}=n} M\left(i_{1}\right) \otimes \cdots \otimes M\left(i_{h}\right) \otimes F\left[S h\left(i_{1}, \ldots, i_{h}\right)\right]\right) \\
& =\bigoplus_{h \geqslant 0} M(h) \otimes_{\Sigma_{h}}\left(M^{\otimes h}\right)=M \circ M
\end{aligned}
$$

La composición de $M \circ M$ representa la primera etapa de composiciones formales y la expresión $M^{\text {oh }}$ puede ser usada para representar h etapas de composiciones formales.

Proposición 4.9 Sean $f: M \rightarrow N$ y $f^{\prime}: M^{\prime} \rightarrow N^{\prime}$ morfismos de $\mathbb{S}-m o ́ d u l o s$, entonces el morfismo dado por $\left(f \circ f^{\prime}\right)\left(x \otimes y_{1} \otimes \cdots \otimes y_{h}\right)=f(x) \otimes f^{\prime}\left(y_{1}\right) \otimes$

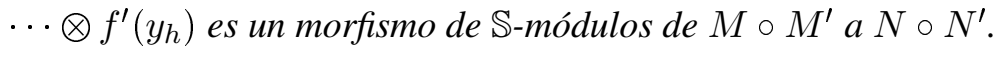

Los $\mathbb{S}$-módulos pueden ser identificados con endofuntores de la categoría DGA-Mod de tal manera que la composición de DGA-módulos coincida con la composición de funtores (ver [2], §5). Este tipo de funtores se llaman funtores de Schur. La siguiente proposición es una consecuencias de esta identificación.

Proposición 4.10 La categoría de $\mathbb{S}$-módulos con la composición o y $I=(0, F, 0, \ldots)$ es una categoría monoidal.

De hecho, los operads simétricos son instancias de monoides sobre la categoría de $\mathbb{S}$-módulos.

Proposición 4.11 Todo operad simétrico determina un monoide en $\mathbb{S}-$ Mod y viceversa.

Demostración. Observe que una aplicación de $\mathbb{S}$-módulos $\eta: I \rightarrow M$ es no cero solamente en aridad 1, entonces determina una aplicación $\eta: F \rightarrow M(1)$ y viceversa. Un morfismo $\mu: M \circ M \rightarrow M$ de $\mathbb{S}$-módulos en aridad $n$ está dado por un morfismo equivariante de DGA-módulos $\mu_{n}$,

$$
\begin{gathered}
\mu_{n}: \bigoplus_{h \geqslant 0} \bigoplus_{i_{1}+\cdots+i_{h}=n} M(h) \otimes_{\Sigma_{h}}\left(M\left(i_{1}\right) \otimes \cdots \otimes M\left(i_{h}\right) \otimes\right. \\
\left.F\left[S h\left(i_{1}, \ldots, i_{h}\right)\right]\right) \rightarrow M(n)
\end{gathered}
$$


el cual está determinado por la colección de morfismos equivariantes,

$$
\gamma: M(h) \otimes \Sigma_{h}\left(M\left(i_{1}\right) \otimes \cdots \otimes M\left(i_{h}\right) \otimes F\left[S h\left(i_{1}, \ldots, i_{h}\right)\right]\right) \rightarrow M(n)
$$

y cada morfismo $\gamma$ está caracterizado como un morfismo

$$
\gamma: M(h) \otimes M\left(i_{1}\right) \otimes \cdots \otimes M\left(i_{h}\right) \rightarrow M(n)
$$

que satisface las condiciones de equivarianza (5) y (6).

Antes de la construcción de operads simétricos libres se necesita una operación más de $\mathbb{S}$-módulos.

Definición 4.12 Sean $M$ y $N$ S-módulos. Se define la suma directa de $M$ y $N$ mediante la fórmula,

$$
(M \oplus N)(n)=M(n) \oplus N(n)
$$

Teorema 4.13 El funtor de olvido $U: \mathcal{O P} \rightarrow \mathbb{S}$-Mod tiene un funtor adjunto a la izquierda $F: \mathbb{S}-M o d \rightarrow \mathcal{O P}$. Al funtor $F$ se le llama el funtor de operads simétricos libres.

Demostración. Sea $M$ un $\mathbb{S}$-módulo. Por la proposición (4.11) solo necesitamos exhibir el operad simétrico libre como un monoide $(F(M), \mu, \eta)$ en $\mathbb{S}$-Mod. Se describirá la construcción de $F(M)$, del producto $\mu$ y el neutro $\eta$, así como la construcción de la unidad y la counidad de la adjunción. Las verificaciones de que estos objetos satisfacen las propiedades requeridas son directas (para más detalles ver $\S 5.4$ en [2]).

Primero, se construye inductivamente para cada $n$ un $\mathbb{S}$-módulo $F(M)_{n}$ de la siguiente manera.

$$
\begin{aligned}
& F(M)_{0}=I \\
& F(M)_{1}=I \oplus M \\
& F(M)_{2}=I \oplus(M \circ(I \oplus M))=I \oplus\left(M \circ F(M)_{1}\right) \\
& F(M)_{n+1}=I \oplus\left(M \circ F(M)_{n}\right)
\end{aligned}
$$

Sea $i_{0}$ la inclusión de $I$ in $F(M)_{1}$. Usando la identidad $M=M \circ I$ y los morfismos $1_{M} \circ i_{0}: M \circ I \rightarrow M \circ F(M)_{1}$ obtenemos el morfismo $i_{1}=$ $1_{I} \oplus\left(1_{M} \circ i_{0}\right): F(M)_{1} \rightarrow F(M)_{2}$. Repitiendo este proceso se obtienen los morfismos,

$$
i_{n}: F(M)_{n} \rightarrow F(M)_{n+1}
$$


definidos por inducción con la fórmula $i_{n+1}=1_{I} \oplus\left(1_{M} \circ i_{n}\right)$. Los $\mathbb{S}$-módulos $F(M)_{n}$ codifican todas las posibles $n$ etapas de composiciones de los elementos de $M$. Para poder colocar junta toda esta información en un solo $\mathbb{S}$-módulo, se toma el colímite sobre el diagrama determinado por los morfismos $i_{j}$.

$$
F(M)=\underset{n}{\operatorname{colim}} F(M)_{n}
$$

El diferencial de $F(M)$ es la extensión evidente del diferencial de $M$.

Ahora necesitamos definir el producto $\mu$ y el neutro $\eta$ para $F(M)$. El neutro está dada por la inclusión $\eta: I \rightarrow F(M)$. El morfismo $\mu: \mathrm{F}(\mathrm{M}) \circ \mathrm{F}(\mathrm{M}) \rightarrow \mathrm{F}(\mathrm{M})$ está determinado por una colección de aplicaciones $\mu_{n, m}: F(M)_{n} \circ F(M)_{m} \rightarrow$ $F(M)_{n+m}$ definidas por inducción sobre $n$, tomando $\mu_{0, m}=1_{F(M)_{m}}$ y para $n>0, \mu_{n, m}$ se define como la composición,

$$
\begin{aligned}
F(M)_{n} \circ F(M)_{m} & =\left(I \oplus M \circ F(M)_{n-1}\right) \circ F(M)_{m} \\
& \cong F(M)_{m} \oplus\left(M \circ F(M)_{n-1}\right) \circ F(M)_{m} \\
& \cong F(M)_{m} \oplus M \circ\left(F(M)_{n-1} \circ F(M)_{m}\right) \\
& \stackrel{1 \oplus 1 \circ \mu_{n-1, m}}{\longrightarrow} F(M)_{m} \oplus M \circ F(M)_{n+m-1} \\
& \stackrel{i+i^{\prime}}{\longrightarrow} F(M)_{n+m}
\end{aligned}
$$

donde $i$ es la inclusión de $F(M)_{m}$ en $F(M)_{n+m}$, y $i^{\prime}$ es la inclusión de $F(M)_{n+m-1}$ como el segundo factor de $F(M)_{n+m}$.

Sea $\mathcal{P}$ un operad simétrico, la counidad $\epsilon: F U \rightarrow 1$ de la adjunción está determinada por los morfismos $\epsilon_{n}: F U(\mathcal{P})_{n} \rightarrow \mathcal{P}$ definidos por inducción de la siguiente manera. $\epsilon_{0}: I \rightarrow \mathcal{P}$ está determinado por la unidad $\eta$ de $\mathcal{P}, \epsilon_{1}=\eta+1$ : $I \oplus U \mathcal{P} \rightarrow \mathcal{P}$ y $\epsilon_{n+1}=\eta+\gamma\left(1 \circ \epsilon_{n}\right): F U(P)_{n}=I \oplus\left(U \mathcal{P} \circ U F(P)_{n}\right) \rightarrow \mathcal{P}$. Finalmente, para $M \in \mathbb{S}$-Mod, la unidad de la adjunción $\eta: 1 \rightarrow U F$, está determinada por las inclusiones en el segundo factor $M \circ F(M)_{n-1} \rightarrow F(M)_{n}$.

En resumen, la adjunción de la proposición 4.13, define para cada operad simétrico $\mathcal{P}$ y $\mathbb{S}$-module $M$, la biyección natural,

$$
\theta: \mathcal{O P}(F(M), \mathcal{P}) \rightarrow \mathbb{S}-\operatorname{Mod}(M, U(\mathcal{P}))
$$

La unidad y la counidad de la adjunción son denotadas $\eta$ y $\epsilon$, respectivamente. Para la unidad $\eta$ tenemos los morfismos,

$$
\begin{aligned}
& \eta: 1_{\mathbb{S}-M o d} \rightarrow U F, \text { y } \\
& \eta_{M}: M \rightarrow U F(M) .
\end{aligned}
$$


Para la counidad $\epsilon$ tenemos,

$$
\begin{aligned}
& \epsilon: F U \rightarrow 1_{\mathcal{O P}}, \mathrm{y} \\
& \epsilon_{\mathcal{P}}: F U(\mathcal{P}) \rightarrow \mathcal{P} .
\end{aligned}
$$

\section{Relaciones entre adjunciones}

Definición 5.1 Un operad no simétrico es definido como un operad simétrico pero sin las acciones de los grupos simétricos. La categoría de los operads no simétricos es denotada $n \mathcal{O P}$.

Definición 5.2 Un $\mathbb{N}$-módulo es un funtor contravariante del grupoide $\mathfrak{N}$ con objetos el conjunto $[0]=\varnothing y[n]=\{1, . ., n\}$ for $n>0, y$ morfismos las aplicaciones identidad, a la categoría DGA-Mod. La categoría de $\mathbb{N}$-módulos se denota $\mathbb{N}$-Mod.

Definición 5.3 Sea $G$ el funtor de olvido de $\mathbb{S}$-Mod a $\mathbb{N}$-Mod.

Teorema 5.4 El funtor de olvido $G: \mathbb{S}-M o d \rightarrow \mathbb{N}$-Mod tiene una adjunta a la izquierda $H: \mathbb{N}$-Mod $\rightarrow \mathbb{S}$-Mod, llamada el funtor libre de $\mathbb{S}$-módulos.

Demostración. Para $M \in \mathbb{N}$-Mod, $H(M)$ es definido como el $\mathbb{S}$-módulo con componentes dadas por $M(n) \otimes F\left[\Sigma_{n}\right]$, para cada $n$. La verificación que satisface las propiedades es directa.

Definición 5.5 Sea $\mathfrak{U}$ el funtor de olvido de la categoría $n \mathcal{O P}$ a $\mathbb{N}$-Mod.

Teorema 5.6 El funtor de olvida $n U: n \mathcal{O P} \rightarrow \mathbb{N}$-Mod tiene una adjunta a la izquierda $n F: \mathbb{N}-$ Mod $\rightarrow n \mathcal{O P}$, llamada el funtor libre de operads no simétricos.

Demostración. El caso no simétrico es similar al caso simétrico, pero sin las consideraciones sobre las acciones de $\Sigma_{n}$. Para un $\mathbb{N}$-módulo $N$, con tal de construir un operad no simétrico libre sobre $N$ necesitamos una suma directa, un producto tensorial y una composición de $\mathbb{N}$-módulos. Estas se definen como,

$$
\begin{aligned}
& (N \oplus E)(n)=N(n) \oplus E(n), \\
& (N \otimes E)(n)=\bigoplus_{i+j=n} N(i) \otimes E(j) \mathrm{y} \\
& (N \circ E)=\bigoplus_{h \geqslant 0} N(k) \otimes E^{\otimes h} .
\end{aligned}
$$


Note que,

$$
N \circ E=\bigoplus_{h \geqslant 0} \bigoplus_{n \geqslant 0} \bigoplus_{i_{1}+\cdots+i_{h}=n} N(h) \otimes E\left(i_{1}\right) \otimes \cdots \otimes E\left(i_{h}\right)
$$

Como en el caso simétrico, los operads no simétricos son monoides sobre la categoría monoidal de $\mathbb{N}$-módulos, donde la estructura monoidal está dada por la composición. Los pasos para la construcción de $n F$ son los mismos que en el caso simétrico.

Definición 5.7 Sea $\mathcal{G}$ el funtor de olvido de la categoría de operads simétricos $\mathcal{O P}$ a la categoría de operads no simétricos $n \mathcal{O P}$.

Teorema 5.8 El funtor $\mathcal{G}: \mathcal{O P} \rightarrow n \mathcal{O P}$ tiene un funtor adjunto a la izquierda $\mathcal{H}: n \mathcal{O P} \rightarrow \mathcal{O P}$.

Demostración. Para un operad no simétrico $\mathcal{P}$ su operad simétrico asociado está dado por $\mathcal{P} \otimes F[\Sigma]$. Las verificaciones son directas.

Teorema 5.9 Las relaciones entre estos funtores de olvido y sus asociados funtores libres, se reúnen en los siguiente diagramas conmutativos.
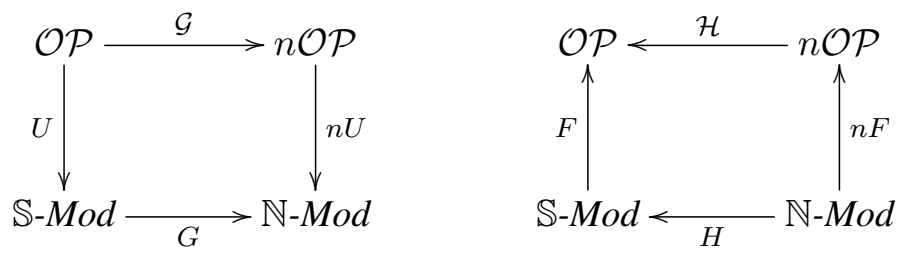

Demostración. La conmutatividad es inmediata por el hecho de que la composición de funtores adjuntos es de nuevo una adjunción, y por la unicidad de la adjunción, sus imágenes son isomorfas.

El diagrama conmutativo de la proposición 5.9 sugiere que se puede usar la construcción de operads no simétricos para describir los operads simétricos libres sobre secuencias simétricas en las cuales las acciones de los grupos simétricos son libres, es decir, para un $\mathbb{S}$-módulo $M$ el operad libre $F(M)$ podría ser interpretado por medio del operad $(\mathcal{H} \circ n F \circ G)(M)$. Lo cual corresponde al caso donde los $\mathbb{S}$-módulos tiene como componentes bar resoluciones $\Sigma_{n}$-libres. Este tipo de $\mathbb{S}$-módulos son usados para describir una $E_{\infty}$-coalgebra estructura en los complejos de cadenas asociados a conjuntos simpliciales (ver [10]).

Definición 5.10 Sea $\Psi: \mathbb{S}-M o d \rightarrow \mathbb{S}-$ Mod el funtor que convierte las acciones por los grupos simétricos de un $\mathbb{S}$-módulo en triviales, es decir,

$$
\Psi(M)(n)=\underset{\sigma \in \Sigma_{n}}{\operatorname{coeq}}(M(n) \underset{\longrightarrow}{\sigma} M(n))
$$


Ejemplo 5.11 Se tiene la siguiente relación entre los operads de los ejemplos $3.4 y 3.5$.

$$
\Psi(U(\mathcal{M}))=U(\mathcal{N})
$$

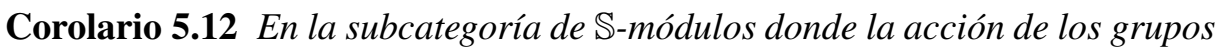
simétricos es libre, la restricción del funtor de operads simétricos libres $F$ es isomorfo al funtor $\mathcal{H} \circ n F \circ G \circ \Psi$.

Demostración. En esta consecuencia del teorema 5.9 es importante notar que, al ser las acciones libres, cada operación abstracta $p$ de $\operatorname{aridad} n$ en $\mathbb{S}$-Mod, tiene asociada una familia de operaciones $p \sigma$, donde $\sigma \in \Sigma_{n}$. El funtor $\Psi$ las identifica como un mismo objeto esta familia y luego la imagen del funtor $\mathcal{H}$ aplicado al resultado de $n F \circ G$, restaura la estructura libre con respecto a las familias $p \sigma$, lo que implica el isomorfismo entre $F$ y $\mathcal{H} \circ n F \circ G \circ \Psi$.

La caracterización dada por el corolario 5.12 es útil a la hora de trabajar operads simétricos libres, debido a que en el caso no simétrico los operads libres pueden ser visualizados fácilmente por árboles planos (ver [4]), como es el caso cuando las acciones sobre los módulos graduados son libres, como en $\operatorname{los} E_{\infty}$-operads.

Ejemplo 5.13 El operad libre $F(U(\mathcal{M}))$ se diferencia del operad $\mathcal{M}$, porque en el primero, $a_{h} \otimes a_{i_{1}} \otimes \cdots \otimes a_{i_{h}}$ no necesariamente coincide con $a_{n}$, donde $n=i_{1}+\cdots i_{h}$. El corolario 5.12 y el ejemplo 5.11 indican que el operad simétrico $F(U(\mathcal{M}))$ se puede describir como $n F \circ G \circ U(\mathcal{N})$ para luego tensorizar por las acciones de los grupos simétricos y hacer las identificaciones necesarias para respetar la equivarianza, lo cual es realizado por el funtor $\mathcal{H}$. Si al único generador $a_{i}$ de la componente $i$ de $G \circ U(\mathcal{N})$ lo interpretamos como un árbol plano con $i$ entradas y 1 salida, los generadores de $n F \circ G \circ U(\mathcal{N})$ son todas las posibles composiciones de estos árboles. Por ejemplo, $a_{7}$ y $a_{2} \otimes a_{3} \otimes a_{4}$ son dos generadores en la componente 7 , donde la segunda se obtiene al conectar en cada entrada de $a_{2}$, una copia de $a_{3}$ y otra de $a_{4}$.

Ejemplo 5.14 Considere el $\mathbb{S}$-módulo $M$ concentrado en aridad 2, dado por el módulo diferencial graduado bar resolución $F\left[\Sigma_{2}\right]$-libre de $F$. El operad libre $F(M)$ es usado en [10] (definición 5.4.3) como punto de partida para construir un $E_{\infty}$-operad con el fin de describir algunas estructuras algebraicas asociadas a generalizaciones de complejos de cadenas. Los elementos de grado $m$ de $M$ son combinaciones lineales de los elementos de la forma $\sigma\left[\sigma_{1}|\ldots| \sigma_{m}\right]$, con $\sigma, \sigma_{1}, \ldots, \sigma_{m} \in \Sigma_{2}$. El borde está dado por $\partial=\sum_{i=0}^{m}(-1)^{i} \partial_{i}$, donde 
$\partial_{0}\left[\sigma_{1}|\ldots| \sigma_{m}\right]=\sigma_{1}\left[\sigma_{2}|\ldots| \sigma_{m}\right], \partial_{i}\left[\sigma_{1}|\ldots| \sigma_{m}\right]=\left[\sigma_{1}|\ldots| \sigma_{i} \sigma_{i+1}|\ldots| \sigma_{m}\right] y$ $\partial_{m}\left[\sigma_{1}|\ldots| \sigma_{m}\right]=\left[\sigma_{1}|\ldots| \sigma_{m-1}\right]$. El único generador en grado cero se escribe []. Con la identificación $F(M) \cong \mathcal{H} \circ n F \circ G \circ \Psi(M)$, los generadores de $F(M)$ son árboles planos cuyos vértices están etiquetados por los elementos de la forma $\left[\sigma_{1}|\ldots| \sigma_{m}\right]$ tensorizados por los grupos simétricos. Por ejemplo, $\tau[]$ en $F(M)$ es representado por el árbol plano con 2 entradas y un vértice etiquetado por [], tensorizado por $\tau$, la permutación no trivial de $\Sigma_{2}$.

\section{Financiamiento}

El autor agradece el financiamiento de la Vicerrectoría de Investigación de la Universidad de Costa Rica a través del proyecto 821-B6-A19.

\section{Referencias}

[1] C. Berger, I. Moerdijk, The Boardman-Vogt resolution of operads in monoidal model categories, Topology 45(2006), no. 5, 807-849. Doi: $10.1016 /$ j.top.2006.05.001

[2] J.L. Loday, B. Vallette, Algebraic operads, Springer, 2012. Doi: $10.1007 / 978-3-642-30362-3$

[3] S. Mac Lane, Categories for the working mathematician, Springer, 1998. Doi: $10.1007 / 978-1-4612-9839-7$

[4] M. Markl, S. Shnider, J.D. Stasheff, Operads in Algebra, Topology and Physics, American Mathematical Society, Providence RI, 2007. In: $10.1090 /$ surv/096

[5] P. May, The geometry of iterated loop space, Springer-Verlag Berlin Heidelberg, 1972. Doi: 10.1007/BFb0067491

[6] A. Prouté, Sur la transformation d'Eilenberg-Mac Lane, C. R. Acad. Sc. Paris 297(1983), 193-194. In: http://163.172.10.123:8080/EilenbergMacLane.pdf

[7] A. Prouté, Sur la diagonale d'Alexander-Whitney, C. R. Acad. Sc. Paris 299(1984), 391-392. In: http://163.172.10.123:8080/AlexanderWhitney.pdf

[8] A. Prouté Introduction à la Logique Catégorique., IMJ-Université Paris 7, 2010. In: https://docplayer.fr/44463391-Introduction-a-la-logiquecategorique.html 
[9] C. Rezk, Spaces of algebra structures and cohomology of operads, Ph.D. thesis, Dept. of Mathematics, Massachusetts Institute of Technology, Cambridge MA, 1996. In: https://dspace.mit.edu/handle/1721.1/41793

[10] J. Sánchez-Guevara, About L-Algebras, Ph.D thesis in Mathematics, Université Paris-Diderot, Paris VII, 2016. In: http://www.theses.fr/2016USPCC204

[11] J.D. Stasheff, Homotopy associativity of H-Spaces. I, American Mathematical Society 108(1963), no. 2, 275-292. Doi: $10.2307 / 1993608$ 